\title{
Infection with Pathogens Transmitted Commonly Through Food and the Effect of Increasing Use of Culture-Independent Diagnostic Tests on Surveillance - Foodborne Diseases Active Surveillance Network, 10 U.S. Sites, 2012-2015
}

\author{
Jennifer Y. Huang, $\mathrm{MPH}^{1}$; Olga L. Henao, $\mathrm{PhD}^{1}$; Patricia M. Griffin, $\mathrm{MD}^{1}$; Duc J. Vugia, $\mathrm{MD}^{2}$; Alicia B. Cronquist, $\mathrm{MPH}^{3}$; Sharon Hurd, MPH${ }^{4}$; \\ Melissa Tobin-D’Angelo, MD ${ }^{5}$; Patricia Ryan, $\mathrm{MD}^{6}$; Kirk Smith, $\mathrm{DVM}^{7}$; Sarah Lathrop, PhD ${ }^{8}$; Shelley Zansky, PhD ; Paul R. Cieslak, MD ${ }^{10}$; \\ John Dunn, DVM ${ }^{11}$; Kristin G. Holt, DVM ${ }^{12}$; Beverly J. Wolpert, $\mathrm{PhD}^{13}$; Mary E. Patrick, $\mathrm{MPH}^{1}$
}

To evaluate progress toward prevention of enteric and foodborne illnesses in the United States, the Foodborne Diseases Active Surveillance Network (FoodNet) monitors the incidence of laboratory-confirmed infections caused by nine pathogens transmitted commonly through food in 10 U.S. sites.* This report summarizes preliminary 2015 data and describes trends since 2012. In 2015, FoodNet reported 20,107 confirmed cases (defined as culture-confirmed bacterial infections and laboratory-confirmed parasitic infections), 4,531 hospitalizations, and 77 deaths. FoodNet also received reports of 3,112 positive culture-independent diagnostic tests (CIDTs) without culture-confirmation, a number that has markedly increased since 2012 (1). Diagnostic testing practices for enteric pathogens are rapidly moving away from culture-based methods. The continued shift from culture-based methods to CIDTs that do not produce the isolates needed to distinguish between strains and subtypes affects the interpretation of public health surveillance data and ability to monitor progress toward prevention efforts. Expanded case definitions and strategies for obtaining bacterial isolates are crucial during this transition period.

FoodNet is a collaboration among CDC, 10 state health departments, the U.S. Department of Agriculture's Food Safety and Inspection Service (USDA-FSIS), and the Food and Drug Administration (FDA). FoodNet conducts active, populationbased surveillance for laboratory-confirmed infections caused by Campylobacter, Cryptosporidium, Cyclospora, Listeria, Salmonella, Shiga toxin-producing Escherichia coli (STEC), Shigella, Vibrio, and Yersinia in 10 sites covering approximately $15 \%$ of the U.S. population (an estimated 49 million persons in 2014). Confirmed infections are defined as culture-confirmed bacterial infections and laboratory-confirmed parasitic infections (e.g., identified by enzyme immunoassay). Positive CIDT results are defined as the detection of antigen or nucleic acid sequences of the pathogen, or for STEC, Shiga toxin or the genes that encode a Shiga toxin, in a stool specimen or enrichment broth using a $\mathrm{CIDT}^{\dagger}$ Positive CIDT results that were confirmed by culture are included only

\footnotetext{
*Connecticut, Georgia, Maryland, Minnesota, New Mexico, Oregon, Tennessee, and selected counties in California, Colorado, and New York (http://www.cdc. gov/foodnet).

$\dagger$ For STEC, only positive CIDT reports that were confirmed at the state public health laboratory were counted.
}

among the confirmed infections. For this analysis, the term "positive CIDT report" refers to positive CIDT results that were not confirmed by culture (either because the specimen was not cultured at the clinical or public health laboratory or because a culture did not yield the pathogen). Hospitalizations occurring within 7 days of specimen collection are recorded. The patient's vital status at hospital discharge, or 7 days after specimen collection if the patient was not hospitalized, is also captured. Hospitalizations and deaths that occur within 7 days of specimen collection are attributed to the infection.

Incidence of confirmed infections is reported for all FoodNet pathogens and calculated by dividing the number of confirmed infections in 2015 by U.S. Census estimates of the surveillance area population for 2014. A second incidence measurement, calculated by adding positive CIDT reports to confirmed infections, is also reported for Campylobacter, Salmonella, Shigella, and STEC. ${ }^{\S}$ A negative binomial model with $95 \%$ confidence intervals (CIs) was used to estimate changes in incidence of infections in 2015 compared with 2012-2014. To describe changes in testing practices, percentage difference in number of positive CIDT reports was calculated for 2015 compared with 2012-2014, by pathogen.

Surveillance for physician-diagnosed postdiarrheal hemolytic uremic syndrome (HUS), a complication of STEC infection, is conducted through a network of nephrologists and infection preventionists and by hospital discharge data review. This report includes HUS data for persons aged $<18$ years for 2014, the most recent year for which data are available, and compares 2014 incidence with 2011-2013 incidence.

\section{Cases of Infection, Incidence, and Trends}

In 2015, FoodNet identified 20,107 confirmed cases of infection, 4,531 hospitalizations, and 77 deaths (Table 1). The number and incidence of confirmed infections per 100,000 population were reported for Salmonella $(\mathrm{n}=7,728$ [incidence $=15.89])$, Campylobacter (6,309 [12.97]), Shigella (2,688 [5.53]), Cryptosporidium (1,612 [3.31]), STEC

\footnotetext{
SIncidence is reported for all STEC serogroups combined because it is not possible to distinguish between O157 and non-O157 based only on a CIDT test for Shiga toxin.
} 
non-O157 (796 [1.64]), STEC O157 (463 [0.95]), Vibrio (192 [0.39]), Yersinia (139 [0.29]), Listeria (116 [0.24]), and Cyclospora (64 [0.13]). Among confirmed infections, the vast majority were diagnosed only by culture; the exception is STEC, for which most were identified by a CIDT (Table 2).

Among 6,827 (88\%) serotyped Salmonella isolates, the top serotypes were Enteritidis, 1,358 (20\%); Newport, 816 (12\%); and Typhimurium, 739 (11\%). Among 175 (91\%) speciated Vibrio isolates, 113 (65\%) were V.parahaemolyticus, 27 (15\%) were $V$. alginolyticus, and 12 (7\%) were $V$. vulnificus. Among

TABLE 1. Number of cases and incidence of confirmed infections, ${ }^{*}$ hospitalizations, and deaths, by pathogen - Foodborne Diseases Active Surveillance Network, United States, $2015^{\dagger}$

\begin{tabular}{|c|c|c|c|c|c|}
\hline \multirow[b]{2}{*}{ Pathogen } & \multicolumn{3}{|c|}{ Cases } & \multirow{2}{*}{$\frac{\text { Hospitalizations }}{\text { No. }(\%)}$} & \multirow{2}{*}{$\frac{\text { Deaths }}{\text { No. (\%) }}$} \\
\hline & No. & Incidence ${ }^{\S}$ & Objective & & \\
\hline \multicolumn{6}{|l|}{ Bacteria } \\
\hline Campylobacter & 6,309 & 12.97 & 8.5 & $1,065(17)$ & $11(0.2)$ \\
\hline Listeria & 116 & 0.24 & 0.2 & $111(96)$ & 15 (12.9) \\
\hline Salmonella & 7,728 & 15.89 & 11.4 & $2,074(27)$ & $32(0.4)$ \\
\hline Shigella & 2,688 & 5.53 & —** & $619(23)$ & $1(0.0)$ \\
\hline STEC 0157 & 463 & 0.95 & 0.6 & $180(39)$ & $3(0.6)$ \\
\hline STEC non-0157 & 796 & 1.64 & 一** & $126(16)$ & $1(0.1)$ \\
\hline Vibrio & 192 & 0.39 & 0.2 & $47(24)$ & $5(2.6)$ \\
\hline Yersinia & 139 & 0.29 & 0.3 & $37(27)$ & $1(0.7)$ \\
\hline \multicolumn{6}{|l|}{ Parasites } \\
\hline Cryptosporidium & 1,612 & 3.31 & -** & $268(17)$ & $8(0.5)$ \\
\hline Cyclospora & 64 & 0.13 & 一** & $4(6)$ & $0(0.0)$ \\
\hline Total & 20,107 & NA & NA & 4,531 & 77 \\
\hline
\end{tabular}

Abbreviations: $\mathrm{NA}=$ not applicable; $\mathrm{STEC}=$ Shiga toxin-producing Escherichia coli.

${ }^{*}$ Confirmed infections are defined as culture-confirmed bacterial infections and laboratory-confirmed parasitic infections.

+ Data for 2015 are preliminary.

$\S$ Per 100,000 population.

9 Healthy People 2020 objective targets for incidence of Campylobacter, Listeria, Salmonella, STEC 0157, Vibrio, and Yersinia infections per 100,000 population.

** No national health objective exists for these pathogens.
$606(76 \%)$ serogrouped STEC non-O157 isolates, the top serogroups were O26 (32\%), O103 (27\%), and O111 (18\%).

Compared with incidence in 2012-2014, the 2015 incidence of confirmed infections was significantly higher for STEC nonO157 (40\% increase; CI $=21 \%-62 \%$ ), and Cryptosporidium ( $57 \%$ increase; $\mathrm{CI}=20 \%-106 \%)$. No significant changes were observed in 2015 for other pathogens compared with the previous 3 -year averages. Among the top three most commonly identified Salmonella serotypes, the incidence in 2015 compared with 2012 2014 was significantly lower for Typhimurium (15\% decrease; $\mathrm{CI}=4 \%-25 \%)$ and unchanged for Enteritidis and Newport.

FoodNet identified 55 cases of postdiarrheal HUS in children $(0.50$ cases per 100,000$)$ in $2014 ; 30(55 \%)$ occurred among children aged $<5$ years $(1.01$ cases per 100,000). Compared with 2011-2013, the incidence was significantly lower for all children ( $27 \%$ decrease; $\mathrm{CI}=1 \%-46 \%)$ but no change for children aged $<5$ years was observed.

FoodNet also received 3,112 positive CIDT reports. The number of positive CIDT reports, by pathogen, were Campylobacter (2,021), Shigella (454), Salmonella (361), and STEC (254). These numbers represent an increase in positive CIDT reports in 2015 of $92 \%$ for Campylobacter, 284\% for Shigella, 247\% for Salmonella, and 120\% for STEC, when compared with the 2012-2014 averages; the overall increase in CIDT reports for these four pathogens was $122 \%$. Adding positive CIDT reports to confirmed cases resulted in the following incidence rates per 100,000 population: 17.12 for Campylobacter, 16.63 for Salmonella, 6.46 for Shigella, and 3.12 for STEC (Figure). Compared with 2012-2014, the 2015 incidence of confirmed infections plus positive CIDT reports was significantly higher for STEC but not for any other pathogen.

TABLE 2. Number and incidence of confirmed infections and positive culture-independent diagnostic test (CIDT) reports, by pathogen, according to culture result - FoodNet, 2015*

\begin{tabular}{|c|c|c|c|c|c|c|}
\hline \multirow[b]{3}{*}{ Pathogen } & \multicolumn{2}{|c|}{ Confirmed infections $^{\dagger}$} & \multicolumn{2}{|c|}{ Positive CIDT reports $§$} & \multirow{2}{*}{\multicolumn{2}{|c|}{$\begin{array}{l}\text { Confirmed infections and } \\
\text { positive CIDT reports }\end{array}$}} \\
\hline & \multirow[b]{2}{*}{$\begin{array}{l}\text { Culture-positive } \\
\text { No. (\%) }\end{array}$} & \multirow{2}{*}{$\begin{array}{c}\text { CIDT-positive and } \\
\text { culture-positive } \\
\text { No. }(\%)\end{array}$} & \multirow{2}{*}{$\begin{array}{c}\text { CIDT-positive and } \\
\text { culture-negative } \\
\text { No. }(\%)\end{array}$} & \multirow{2}{*}{$\begin{array}{l}\text { CIDT-positive and } \\
\text { no culture } \\
\text { No. }(\%)\end{array}$} & & \\
\hline & & & & & No. & $\begin{array}{c}\text { Incidence per } \\
100,000 \text { population }\end{array}$ \\
\hline Campylobacter & $5,964(72)$ & $345(4)$ & $851(10)$ & $1,170(14)$ & 8,330 & 17.12 \\
\hline Salmonella & $7,354(91)$ & $374(5)$ & $141(2)$ & $220(3)$ & 8,089 & 16.63 \\
\hline Shigella & $2,567(82)$ & $121(4)$ & $160(5)$ & $294(9)$ & 3,142 & 6.46 \\
\hline STEC & $55(4)$ & $1,204(80)$ & $111(7)$ & $143(9)$ & 1,513 & 3.12 \\
\hline Vibrio & $190(95)$ & $2(1)$ & $7(3)$ & $2(1)$ & 201 & 0.41 \\
\hline Yersinia & $137(90)$ & $2(1)$ & $3(2)$ & $10(7)$ & 152 & 0.31 \\
\hline Listeria & $116(100)$ & $0(0)$ & $0(0)$ & $0(0)$ & 116 & 0.23 \\
\hline Total & 16,383 & 2,048 & 1,273 & 1,839 & 21,543 & NA \\
\hline
\end{tabular}

Abbreviations: NA = not applicable; STEC = Shiga toxin-producing Escherichia coli.

* Data for 2015 are preliminary.

† Confirmed infections are defined as culture-confirmed bacterial infections and laboratory-confirmed parasitic infections.

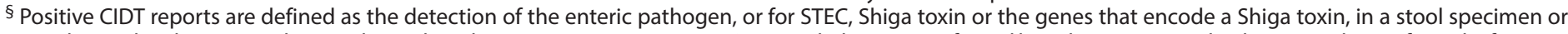

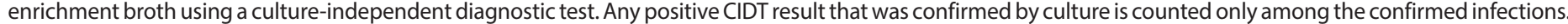
For STEC, only positive CIDT reports that were confirmed at the state public health laboratory were counted.

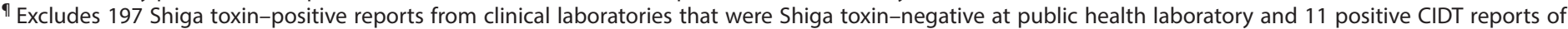
detection of $\mathrm{O} 157$ antigen without testing for Shiga toxin. 


\section{Discussion}

Use of CIDTs is finding cases that were not being previously diagnosed. Among confirmed cases, the incidence of Cryptosporidium and STEC non-O157 infections in 2015 was significantly higher than the average for the previous 3 years. The increase in incidence of STEC non-O157 infections is attributable, in part or in full, to increases in diagnostic testing (2). The proportion of laboratories testing for STEC nonO157 increased to $74 \%$ in 2015 , compared with $55 \%$ in 2012 (FoodNet, unpublished data). The increase in Cryptosporidium follows the pattern observed in national data since 2005 and is likely also driven by increases in diagnostic testing $(3,4)$.

The incidence of Salmonella serotype Typhimurium infections continues to decline, and it has dropped to the third most commonly reported serotype. The use of a live attenuated Typhimurium vaccine in poultry (5), in addition to more stringent performance standards for Salmonella contamination of poultry carcasses (G) might have contributed to this decline. The significant decrease in HUS incidence in 2014 compared with the preceding 3 years (2011-2013) mirrors significant decreases in STEC O157 incidence observed during the same period (7). Efforts are still needed to decrease contamination of produce, beef, and other foods to achieve the Healthy People 2020 goal for STEC O157 of 0.6 cases per 100,000 population.9

The percentage of infections diagnosed only by CIDTs markedly increased in 2015. Diagnostic testing practices for enteric pathogens are rapidly moving away from culture-based methods, and the impact of this change varies by pathogen. Although CIDTs are still most commonly being used for Campylobacter and STEC, the highest percentage increase in use compared with the previous 3-year average was observed for Shigella and Salmonella, most likely due to laboratories using the newly available DNA-based syndrome panels (FoodNet, unpublished data)

In FoodNet, current methods to assess trends in the incidence of illness caused by bacterial pathogens are based only on cultureconfirmed infections. The ability to assess and interpret change is impeded as the number of positive CIDT reports continues to rise because of important limitations in the understanding of CIDTs and possible changes in clinician and laboratory practices surrounding them. For example, analyses need to consider the likelihood of false-positive CIDTs and of CIDTs that are more

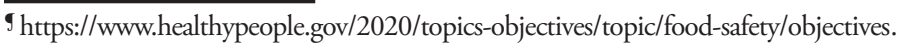

sensitive than routine culture methods; such characteristics vary among CIDTs. The availability of CIDTs might also increase testing for some pathogens. Surveillance systems need to adapt to these changes by expanding case definitions to include positive CIDT reports. Isolates are still needed for antimicrobial susceptibility testing, serotyping, subtyping, and whole genome sequencing (1); these data are critical for monitoring trends, detecting clusters of illness, and investigating outbreaks. For Salmonella, with serotypes diverse in reservoirs and sources, the inability to distinguish serotypes will prevent tracking of important changes in incidence by serotype, and markedly limit detection and investigation of outbreaks. For STEC, because identification of serogroups requires culture, it is not known which STECpositive CIDT reports represent O157 versus non-O157.

The findings in this report are subject to at least five limitations. First, increasing use of CIDTs by clinical laboratories might affect the number of culture-confirmed infections reported; use of CIDTs might result in an increase (as seen for STEC nonO157 infections) or decrease (as fewer cases might be diagnosed by traditional methods) in reported incidence. Second, the sensitivity and specificity of CIDTs vary by test type, brand, and other factors; some CIDT reports could be false positives (1). Third, health care-seeking behaviors, access to health services, and other characteristics of the population in the surveillance 


\section{Summary}

What is already known about this topic?

The incidence of infections transmitted commonly by food has remained largely unchanged for many years. Multifaceted approaches involving public health, regulatory agencies, industry, and consumers are required to reduce the incidence.

What is added by this report?

Compared with average incidence in 2012-2014, in 2015, the incidence of Cryptosporidium and non-O157 STEC infections was higher and might, in part, be caused by the use of cultureindependent diagnostic tests (CIDTs), which more than doubled during the comparison period.

What are the implications for public health practice?

Some information about the bacteria causing infections, such as subtype and antimicrobial susceptibility, can only be obtained if a CIDT-positive specimen is also cultured. Increasing use of CIDTs affects the interpretation of public health surveillance data and the ability to monitor progress towards prevention efforts. Currently, reflex culturing of specimens with positive CIDT reports should be considered for bacterial pathogens to obtain isolates needed for public health practice. In the long term, expedited research and development are needed to create methods to detect the genetic sequences of pathogens directly and rapidly from stool specimens, which could also benefit clinical and public health practice because subtype, resistance profile, and other features can be obtained from the genetic sequence.

area might affect the generalizability of the findings. Fourth, the proportion of illnesses transmitted by non-food routes differs by pathogen; data provided in this report are not limited to infections from food. ${ }^{* *}$ Finally, changes in incidence between periods can reflect year-to-year variation during those periods rather than sustained trends, and the number of infections and patterns observed might change as final data become available.

The use of CIDTs in clinical laboratories has many advantages. Illnesses can be diagnosed much faster than when culture is required. Also, some CIDTs are becoming available to detect infections caused by pathogens not routinely sought by standard laboratory methods. One of these is enterotoxigenic E. coli, an important cause of travelers' diarrhea (8).

More work is needed to extend the benefits of CIDT to the public health sector. During this initial period when clinical laboratories are transitioning to the use of CIDTs, reflex culturing ${ }^{\dagger \dagger}$ of specimens with positive CIDT reports should be considered for bacterial pathogens to obtain isolates needed

\footnotetext{
** FoodNet's data is used to support Interagency Food Safety Analytics Collaboration's projects to identify foods that are important sources of illnesses. http://www.cdc.gov/foodsafety/ifsac/index.html.

$\dagger^{\dagger}$ Reflex culturing for bacterial pathogens is culturing specimens with positive CIDT results
}

for public health practice. For the future, expedited research and development are needed to create methods to detect the genetic sequences of pathogens directly and rapidly from stool specimens, which has the potential to benefit both clinical and public health practice, because subtype, resistance profile, and other features can be obtained from the genetic sequence.

\section{Acknowledgments}

Workgroup members, Foodborne Diseases Active Surveillance Network (FoodNet), Emerging Infections Program; Staci Dixon, Robert V. Tauxe, the Health Communications Team, Division of Foodborne, Waterborne, and Environmental Diseases, National Center for Emerging and Zoonotic Diseases, CDC.

\footnotetext{
${ }^{1}$ Division of Foodborne, Waterborne, and Environmental Diseases, National Center for Emerging and Zoonotic Infectious Diseases, CDC; ${ }^{2}$ California Department of Public Health; ${ }^{3}$ Colorado Department of Public Health and Environment; ${ }^{4}$ Connecticut Department of Public Health; ${ }^{5}$ Georgia Department of Public Health; ${ }^{6}$ Maryland Department of Health and Mental Hygiene; ${ }^{7}$ Minnesota Department of Health; ${ }^{8}$ University of New Mexico, Alburquerque; ${ }^{9} \mathrm{New}$ York State Department of Health; ${ }^{10}$ Oregon Health Authority; ${ }^{11}$ Tennessee Department of Health; ${ }^{12}$ Food Safety and Inspection Service, U.S. Dept of Agriculture; ${ }^{13}$ Center for Food Safety and Applied Nutrition, Food and Drug Administration.
}

Corresponding author: Mary Patrick, mepatrick@cdc.gov, 404-639-3433.

\section{References}

1. Iwamoto M, Huang JY, Cronquist $A B$, et al. Bacterial enteric infections detected by culture-independent diagnostic tests-FoodNet, United States, 2012-2014. MMWR Morb Mortal Wkly Rep 2015;64:252-7.

2. Gould LH, Mody RK, Ong KL, et al.; Emerging Infections Program Foodnet Working Group. Increased recognition of non-O157 Shiga toxin-producing Escherichia coli infections in the United States during 2000-2010: epidemiologic features and comparison with E. coli O157 infections. Foodborne Pathog Dis 2013;10:453-60. http://dx.doi. org/10.1089/fpd.2012.1401

3. Painter JE, Hlavsa MC, Collier SA, Xiao L, Yoder JS. Cryptosporidiosis surveillance-United States, 2011-2012. MMWR Surveill Summ 2015;64(No. SS-03):1-14.

4. Robinson TJ, Cebelinski EA, Taylor C, Smith KE. Evaluation of the positive predictive value of rapid assays used by clinical laboratories in Minnesota for the diagnosis of cryptosporidiosis. Clin Infect Dis 2010;50:e53-5. http://dx.doi.org/10.1086/651423

5. Desin TS, Köster W, Potter AA. Salmonella vaccines in poultry: past, present and future. Expert Rev Vaccines 2013;12:87-96. http://dx.doi. org/10.1586/erv. 12.138

6. US Department of Agriculture Food Safety Inspection Service. New performance standards for Salmonella and Campylobacter in young chicken and turkey slaughter establishments: new compliance guides. Washington, DC: US Department of Agriculture, Food Safety Inspection Service; 2010. http://www.fsis.usda.gov/OPPDE/rdad/FRPubs/2009-0034.pdf

7. Crim SM, Griffin PM, Tauxe R, et al. Preliminary incidence and trends of infection with pathogens transmitted commonly through foodFoodborne Diseases Active Surveillance Network, 10 U.S. sites, 20062014. MMWR Morb Mortal Wkly Rep 2015;64:495-9.

8. Medus C, Besser JM, Juni BA, et al. Long-term sentinel surveillance for enterotoxigenic $E$. coli and non-O157 Shiga toxin-producing $E$. coli in Minnesota. Open Forum Infect Dis. Epub February 21, 2016. http:// dx.doi.org/10.1093/ofid/ofw003 